\title{
One-pot synthesis of dextran-coated iron oxide nanoclusters for real-time regional lymph node mapping
}

This article was published in the following Dove Press journal:

International Journal of Nanomedicine

27 April 2017

Number of times this article has been viewed

\author{
Chaoping $\mathrm{Fu}^{1, *}$ \\ Haipeng Zhou ${ }^{2, *}$ \\ Yanan Wang ${ }^{2, *}$ \\ Dong $\mathrm{Liu}^{2}$ \\ Junmeng $\mathrm{Li}^{2}$ \\ Haijun Deng ${ }^{2}$ \\ Xiaolong $\mathrm{Qi}^{2}$ \\ Tao Chen ${ }^{2}$ \\ Li-Ming Zhang' \\ Guoxin $\mathrm{Li}^{2}$ \\ 'PCFM Lab and GDHPPC Lab, School \\ of Materials Science and Engineering, \\ Sun Yat-sen University, ${ }^{2}$ Department \\ of General Surgery, Nanfang Hospital, \\ Southern Medical University, \\ Guangzhou, People's Republic of \\ China \\ *These authors contributed equally \\ to this work
}

\begin{abstract}
The intraoperative precision cleaning of lymph nodes (LNs) is an essential component of treating neoplastic disease. To develop efficient probes for the targeted detection of LNs that could act as carriers for the specific diagnosis and treatment of metastatic LNs in the future, dextran-coated iron oxide nanoclusters (DIONs) were synthesized using a one-pot coprecipitation procedure. These modified DIONs have good water dispersibility, cytocompatibility, an optimum size, and a stable, dark brown color for LN imaging. In this study, cytotoxicity was evaluated using lymphatic endothelial cells (LECs) to predict biosafety and biocompatibility. Most importantly, the effectiveness of DIONs in mapping perigastric LNs in Sprague Dawley rats following injection into the gastric submucosal layer was demonstrated. In addition, a longterm tracing in vivo (from 4 days to 3 months) indicated that the DIONs had good biosafety and biocompatibility according to an evaluation of the behavior and blood biochemistry of the rat and a histopathological examination of the important organs.
\end{abstract}

Keywords: dextran-coated iron oxide nanoclusters, lymph node mapping, biosafety, lymphatic endothelial cells

\section{Introduction}

The lymphatic system plays an important role in the spread of cancer cells, and lymph node (LN) metastasis is a universal sign of tumor progression, such as in gastrointestinal cancer, esophageal cancer, and breast cancer. ${ }^{1}$ Curative surgical resection is the only method for controlling these diseases. To decrease the mortality and morbidity rates, the inclusion of only appropriate candidates for surgical procedures and the selection of the appropriate LNs for dissection are mandatory. ${ }^{2}$ For these reasons, correct intraoperative staging of metastatic LNs is essential for developing an individualized surgery. ${ }^{3-5}$

Currently, the assessment of regional metastatic LNs is usually conducted via an imagological diagnosis, such as computed tomography (CT), magnetic resonance imaging (MRI) and 18F-fluoro-2-deoxyglucose positron emission tomography (FDGPET). These methods, especially FDG-PET, have exhibited usefulness in the diagnosis of primary tumors and regional metastatic LNs. However, because of the limitations of the equipment, intraoperative mapping could not be performed with these imaging methods, possibly leading to inadequate removal of metastatic LNs or excessive LN dissection. ${ }^{6-10}$ In addition, certain dyes or isotopes have been used primarily to provide intraoperative visual guidance in mapping regional LNs, but these materials have no tumor specificity, which could cause a high false-negative rate for metastatic LNs. ${ }^{11}$ 
Carbon nanoparticles (CNPs), a type of dye widely used in the clinic to map LNs, can improve LN retrieval in carcinoma cases. ${ }^{12}$ In a previous study, CNPs were injected into the tumor submucosal or subserosal layer 1 day before surgery, and the particles then quickly entered into the lymphatic vessels instead of the bloodstream because of their diameter limits (100-200 nm). The CNPs gathered together in LNs, thereby labeling the nodes black to clearly map regional LNs. Even though CNPs have a high degree of lymphatic targeting, they are like other dyes that have no tumor specificity, and most of the time, CNPs can only be used to improve the rate of LN detection and shorten detection time. ${ }^{4}$

Therefore, we chose to synthesize modifiable polymeric nanoparticles that not only possess a high degree of lymphatic targeting but can also carry fluorescent material for intraoperative metastatic LN mapping. To address these issues, in this research, we first designed a dextran-coated iron oxide nanocluster (DION), which mainly consists of $\mathrm{Fe}^{3+} / \mathrm{Fe}^{2+}$ and dextran. The diameter of the DIONs was limited to 100-200 nm, similar to CNPs, so that the DIONs will specifically gather together within LNs. Furthermore, because the modifiable dextran contains a large number of hydroxyl groups, which can be conjugated to drugs or fluorescent material either by direct attachment or through a linker, there exists a potential application in the specific diagnosis and treatment of metastatic LNs. ${ }^{13-16}$ Therefore, the main purpose of this study is to evaluate the effectiveness of DIONs in regional LN mapping and the biosafety of these nanoparticles after injection, thereby laying a foundation for further clinical use and functionalized modifications.

\section{Materials and methods Materials}

Dextran was purchased from Thermo Fisher Scientific Inc. (Waltham, MA, USA); iron (III) chloride, iron (II) chloride and ammonium hydroxide were purchased from Energy Chemical Inc. (Shanghai, China) and CNPs were purchased from Chongqing LUMMY Pharmaceutical Co. (Chongqing, People's Republic of China). All other chemicals used were of analytical grade.

\section{Rats and cells}

Female Sprague Dawley rats aged 6-8 weeks were purchased from the Central Laboratory of Animal Science at Southern Medical University (Guangzhou, China). The rats had been maintained at the Laboratory Animal Centre of Nanfang hospital in a specific pathogen-free environment. All animal experiments in this study were approved by, and followed the protocols (Application No NFYY-2015-114) of the Nanfang hospital animal ethics committee, Southern Medical University (Guangzhou, China). Human lymphatic endothelial cell (LEC) lines were purchased from Cellbio (Shanghai, China). The cells were cultured in high-sugar DMEM (HyClone, Logan, UT, USA) with $10 \%$ fetal bovine serum (FBS; Thermo Fisher Scientific), $8 \mathrm{mg} / \mathrm{mL}$ penicillin and $8,000 \mathrm{U} / \mathrm{mL}$ streptomycin at $37^{\circ} \mathrm{C}$ in a humidified $5 \% \mathrm{CO}_{2}$ incubator.

\section{Preparation of DIONs}

DIONs were synthesized using a one-pot coprecipitation procedure according to the protocol described by Kamat et al. ${ }^{17} \mathrm{FeCl}_{3} \cdot 6 \mathrm{H}_{2} \mathrm{O}$ and $\mathrm{FeCl}_{2} \cdot 4 \mathrm{H}_{2} \mathrm{O}$ at an $\mathrm{Fe}^{3+} / \mathrm{Fe}^{2+}$ molar ratio of 1.8:1 and dextran (3.0 g, low fraction, MW 60-90 kDa) were dissolved in $20 \mathrm{~mL}$ of water. The mixture was cooled to $0^{\circ} \mathrm{C}-4^{\circ} \mathrm{C}$, and the solution was filtered through a $0.22 \mu \mathrm{m}$ membrane with continuous stirring under an argon atmosphere, and then, $0.5 \mathrm{~mL}$ of ice-cold $25 \% \mathrm{NH}_{4} \mathrm{OH}$ was added dropwise to neutralize the reaction mixture, which was then maintained at $0^{\circ} \mathrm{C}-4^{\circ} \mathrm{C}$ for $15 \mathrm{~min}$. Subsequently, the reaction mixture was heated to $85^{\circ} \mathrm{C}-90^{\circ} \mathrm{C}$ and maintained at this temperature for $2 \mathrm{~h}$, leading to a stable, dark brown dextran-coated iron oxide solution. The reaction mixture was cooled to room temperature, and the excess dextran and $\mathrm{NH}_{4} \mathrm{OH}$ were removed by dialysis against double-distilled water under a nitrogen atmosphere. Using ultrafiltration, the resulting solution was filtered through a $0.22 \mu \mathrm{m}$ membrane to obtain the DIONs. The Fourier transform infrared (FTIR) spectra were obtained using a Nexus 670 model FTIR spectrophotometer (Thermo Nicolet Corporation, Madison, WI, USA) at a $4 \mathrm{~cm}^{-1}$ resolution using $\mathrm{KBr}$ pellets. The particle size distribution and morphology of the DIONs in phosphate buffered saline (PBS, $\mathrm{pH}=7.4$ ) were characterized through dynamic light scattering (DLS; Brookhaven, Upton, NY, USA), and transmission electron microscopy (TEM) images were obtained using a JEM-2010HR transmission electron microscope (JEOL Ltd., Tokyo, Japan).

\section{In vivo mapping of $L N s$}

For this experiment, rats underwent preoperative fasting and water deprivation for $24 \mathrm{~h}$. A rat was randomly selected, and after exposing the abdominal wall, local disinfection was carried out 3 times by iodophor, and 1\% pentobarbital sodium $(0.4 \mathrm{~mL} / 100 \mathrm{~g}$ weight $)$ was injected into the rat's abdominal cavity. After the administration of anesthesia and certain routine preoperative preparations, rats were divided into 4 groups ( 3 rats in each group), and then different concentrations (25, 50,75 and $100 \mathrm{mg} / \mathrm{mL}$ ) of DIONs were injected into the rat's gastric submucosal layer at 5 points $(0.05 \mathrm{~mL}$ for each point) for $24 \mathrm{~h}$ to explore an appropriate concentration for the 
next step experiment. Then, 24 rats were regularly fed and randomly divided into 8 groups (3 rats per group). At 6, 12, $18,24,30,36,42$ and $48 \mathrm{~h}$ after injection of the applicable concentration of DIONs, the rat's abdomen was exposed and inspected for any complication, such as acute inflammatory exudation, gastric perforation or adhesive intestinal obstruction, etc. Then the stained LNs were recorded using a Canon digital camera and statistically analyzed.

After the in vivo observation, the rats were euthanized with a lethal dose of pentobarbital, and all the stained perigastric LNs were harvested. These LNs were then fixed in 10\% neutral buffered formalin. All the samples were then serially sectioned at $40 \mu \mathrm{m}$ intervals and stained with hematoxylin and eosin $(\mathrm{H} \& \mathrm{E})$. To further validate the existence of the DIONs in LNs, microscopy images of Prussian blue-stained tissue slices were obtained, which revealed the presence of iron in the LNs of the DION-injected rat. ${ }^{1}$

\section{In vitro cytotoxicity and proliferation assay}

The cytotoxicity and proliferation of DIONs in LECs were assessed with a cell counting kit (CCK)-8 assay, and the cells coincubated with DIONs were abbreviated as DIONs-LECs. Cells were seeded into 96-well plates at a density of 2,000 cells per well and cultured for $24 \mathrm{~h}$ in high-sugar DMEM plus $10 \%$ FBS at $37^{\circ} \mathrm{C}$ and $5 \% \mathrm{CO}_{2}$. Then, the cells were incubated for 5 days with DIONs at different concentrations $(0.5,1.0,1.5$ and $2.0 \mathrm{mg} / \mathrm{mL}$ ) as the DIONs-LECs group and without DIONs as the LECs group. In addition, for the blank control group, there was only high-sugar DMEM plus 10\% FBS in the plates without any cells. Then, the cells were quantified at various times $(0$, 6, 18, 24, 48, 72 and $96 \mathrm{~h}$ ) using the CCK-8 assay (Dojindo, Kumamoto, Japan) and a microplate reader (BioTek, Winooski, VT, USA). The absorbance data (optical density [OD] value) at a wavelength of $450 \mathrm{~nm}$ were collected to analyze cytotoxicity and proliferation. Cell viability was used to analyze the cytotoxicity. In addition, the proliferation was evaluated by comparing the DIONs-LECs group's OD value with that in the LECs group. This experiment was repeated 3 times. The following is the calculation formula of cell viability:

$$
\text { Cell viability }(\%)=\left(\mathrm{OD}_{1}-\mathrm{OD}_{0}\right) /\left(\mathrm{OD}_{2}-\mathrm{OD}_{0}\right) \times 100 \%
$$

In the calculation formula: $\mathrm{OD}_{1}$ refers to the $\mathrm{OD}$ value for the DIONs-LECs group, $\mathrm{OD}_{2}$ refers to the $\mathrm{OD}$ value for the LECs group and $\mathrm{OD}_{0}$ refers to the OD value for the blank control group.

\section{In vitro coincubation of LECs and DIONs}

To further analyze the influence on morphology at the cellular level, additional experiments were performed by incubating
DIONs with LECs. First, Prussian blue staining for iron detection was performed to verify the presence of the DIONs in cells. DIONs were added to high-sugar DMEM plus $10 \%$ FBS to 4 final concentrations of $0.5,1.0,1.5$ and $2.0 \mathrm{mg} / \mathrm{mL}$ and coincubated with $5.0 \times 10^{5}$ LECs for $24 \mathrm{~h}$ as the DIONsLECs group, and then the DIONs-LECs were taken, and the culture medium was removed. They were rinsed with PBS to remove the extracellular free iron particles. After fixing with $4 \%$ paraformaldehyde for $10 \mathrm{~min}$, they were washed twice with double-distilled water. Then, they were incubated in $2 \%$ potassium ferrocyanide and $6 \%$ aqueous hydrochloric acid at $37^{\circ} \mathrm{C}$ and $5 \% \mathrm{CO}_{2}$ for $30 \mathrm{~min}$, and washed 3 times with double-distilled water. Nuclear fast red was used to restain the nucleus for $5 \mathrm{~min}$. They were washed with doubledistilled water 3 times and then observed by fluorescence microscopy (IX-70, Olympus Corporation, Tokyo, Japan). ${ }^{18}$ Blue particles in the cell indicated success of staining. Then, we chose the DIONs with an appropriate concentration that had a satisfactory blue staining effect for the following comparison. Finally, we compared the DIONs-LECs' morphology, size and proliferation condition with normal LECs and repeated the experiment 3 times.

\section{In vivo biodistribution evaluation}

In this experiment, we conjugated DIONs with $\mathrm{Cy} 5$ dye (Amersham Pharmacia Biotech UK Ltd. Locations, Little Chalfont, UK). The resultant DIONs were abbreviated as DIONs-Cy5. DIONs-Cy5 were prepared through Cy5dextrans, and were prepared in a similar way to a previously described protocol. ${ }^{19}$ Near-infrared dye Cy5 was applied as the fluorescent probe. Then, $75 \mathrm{mg} / \mathrm{mL}$ DIONs-Cy5 was injected into 4 rats' gastric submucosal layers at 5 points $(0.05 \mathrm{~mL}$ per point). After the operation, the animals were sacrificed at 6 , 12, 24 and $48 \mathrm{~h}$; perigastric LNs and major organs, including gastric organs and the liver, kidney, lung, spleen and heart, were excised and imaged with a small animal in vivo fluorescence imaging system (Carestream In-Vivo Imaging System FXPRO; Carestream Health, Inc., Woodbridge, CT, USA). This experiment was repeated 3 times.

\section{In vivo biosafety evaluation}

The remaining 54 rats were randomly divided into the following 3 groups (18 rats per group): group I (injected with DIONs), group II (injected with CNPs) and group III (the control group, injected with saline). Behavioral changes and the medicinal residue around the injection point were assessed at predetermined time points ( 4 days, 1 week, 2 weeks, 1 month, 2 months and 3 months). In addition, using a standard blood collection technique, $1 \mathrm{~mL}$ of blood was drawn from the 
postcava and separated by centrifugation into cellular and plasma fractions for biochemical examination. Upon completion of the blood collection, the rats were sacrificed with a lethal dose of pentobarbital. The major organs (liver, kidney, spleen, heart and brain) in 3 groups of rats were harvested, fixed in 4\% neutral buffered formalin, embedded routinely into paraffin, and stained with $\mathrm{H} \& \mathrm{E}$, and the histopathological lesions were examined and evaluated.

The following experiment was to test the blood iron content. The other 36 rats were regularly fed and randomly divided into the following 2 groups: DIONs group (injected with DIONs, 18 rats) and control group (normal rats, 18 rats). Before this experiment, rats underwent preoperative fasting and water deprivation for $24 \mathrm{~h}$. After the administration of anesthesia and certain routine preoperative preparations as mentioned earlier, $75 \mathrm{mg} / \mathrm{mL}$ of DIONs were injected into the DIONs group rats' gastric submucosal layer at 5 points ( $0.05 \mathrm{~mL}$ for each point). At $6,12,24,48,96$ and $168 \mathrm{~h}$ after injection, the abdomen of the rats of the 2 groups was exposed (3 rats for each time point), and using a standard blood collection technique, $6 \mathrm{~mL}$ of blood was drawn from the abdominal aorta for testing the blood iron content by atomic absorption spectrometry (AAS). ${ }^{20}$ Then, the blood iron content of the 2 groups was compared. After the experiment, the rats were euthanized with a lethal dose of pentobarbital.

\section{Statistical analysis}

Data are expressed as mean \pm SD and were analyzed by SPSS 22.0 statistic software. All experiments were repeated at least 3 times with comparable results, unless indicated otherwise. Statistical evaluation of the data was performed using the unpaired Student's $t$-test and analysis of variance (ANOVA) followed by Scheffe's post hoc test. Significant differences were considered when $P<0.05$.

\section{Results and discussion \\ Structural characterization of DIONs}

The DIONs were prepared by coprecipitating $\mathrm{Fe}^{3+}$ and $\mathrm{Fe}^{2+}$ salts in the presence of dextran $(60-90 \mathrm{kDa})$, as shown in Figure 1A. The DLS data showed that the as-prepared DIONs had a hydrodynamic diameter of $165 \mathrm{~nm}$ (Figure 1B) and a zeta potential of $-33.4 \mathrm{mV}$. Furthermore, Figure $1 \mathrm{C}$ gives the FTIR spectra of $\mathrm{Fe}_{3} \mathrm{O}_{4}$, dextran and DIONs. In comparison with the spectrum of $\mathrm{Fe}_{3} \mathrm{O}_{4}$ without any modification, the spectrum of DIONs showed not only the characteristic bands of $\mathrm{Fe}_{3} \mathrm{O}_{4}$ at $570 \mathrm{~cm}^{-1}$ (attributed to $\mathrm{Fe}-\mathrm{O}$ stretching) but also the characteristic band of dextran group at 1,156 and 2,934 $\mathrm{cm}^{-1}$ (attributed to $\mathrm{C}-\mathrm{O}$ stretching and $\mathrm{C}-\mathrm{H}$ stretching), which could confirm that the surface of DIONs was coated by dextran. To understand how the DIONs are packed, samples were studied by TEM. Figure 1D shows that the DION nanocrystals are largely present as isolated clusters of iron oxide nanoparticles with dense packing. The TEM images indicated that the DIONs had a diameter of $\sim 150 \mathrm{~nm}$ (Figure 1E), a very appropriate particle size for specific LN mapping. The dextran coating is not visible because this material does not significantly attenuate electron beams in TEM. These modified DIONs have good water dispersibility and magnetic properties and a stable, dark brown color, as illustrated in Figure 1F. All these results indicated that DIONs could be successfully fabricated with a proper particle size and superior stability for LN mapping.

\section{In vivo $L N$ mapping}

For the in vivo experiments, Sprague Dawley rats were injected with DIONs to evaluate the capacity of these particles to map LNs. After the administration of anesthesia and certain routine preoperative preparations as mentioned earlier, different concentrations $(25,50,75$ and $100 \mathrm{mg} / \mathrm{mL})$ of DIONs were injected into the rats' gastric submucosal layer at 5 points $(0.05 \mathrm{~mL}$ per point $)$ to determine a concentration that had a satisfactory effect and could be applied to mapping. At $24 \mathrm{~h}$ after injection, the results indicate that $75 \mathrm{mg} / \mathrm{mL}$ DIONs had a satisfactory labeling effect (Figure 2A). Therefore, $75 \mathrm{mg} / \mathrm{mL}$ of DIONs was chosen for injection to evaluate the labeling effect at different time points (from 6 to $48 \mathrm{~h}$ ). The results revealed that stained LNs gradually appeared at $6 \mathrm{~h}$ and could be clearly identified with the naked eye at $24 \mathrm{~h}$ after injection, and the number of stained LNs is shown in Figure $2 \mathrm{~B}$. The statistical results showed that the number of the stained LNs at $24 \mathrm{~h}\left(4.33 \pm 1.53,{ }^{*} P<0.05\right)$ was more than the number at other times. In addition, comparison of numbers at different times indicated statistically significant differences $(F=3.354, P<0.05)$. The color then slowly faded in the next $24 \mathrm{~h}$ (Figure 2C). After taking the photo, all the stained perigastric LNs were harvested for Prussian blue-stained tissue slices to further validate the presence of DIONs in LNs. It is known ${ }^{21}$ that Prussian blue staining can give a characteristic blue color in the presence of ferric ions. As seen, tissue at $6 \mathrm{~h}$ postinjection showed no obvious blue stains, while the $24 \mathrm{~h}$ postinjection led to blue color inside the LNs (Figure 2D). These results confirmed further the specific uptake of DIONs by LNs. In conclusion, all these results suggested that $75 \mathrm{mg} / \mathrm{mL}$ of DIONs injected into the submucosal 
A
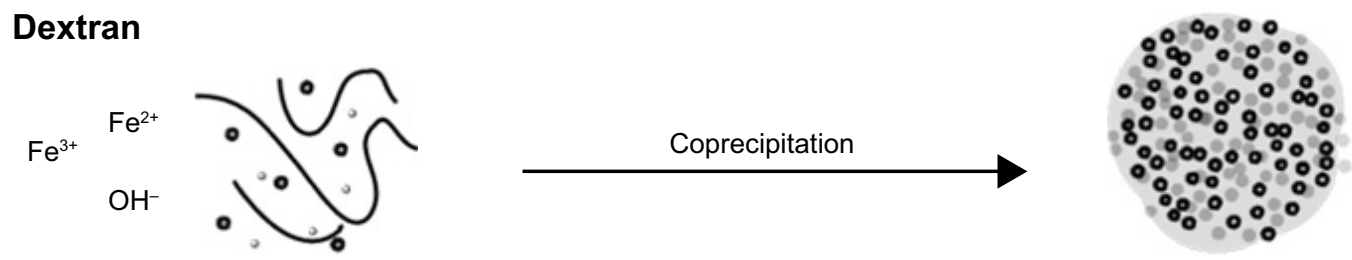

DIONs

B

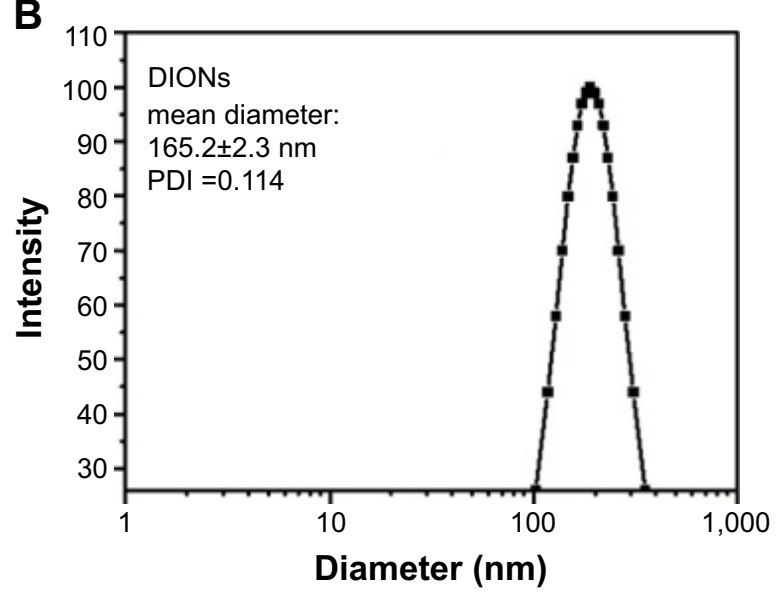

D

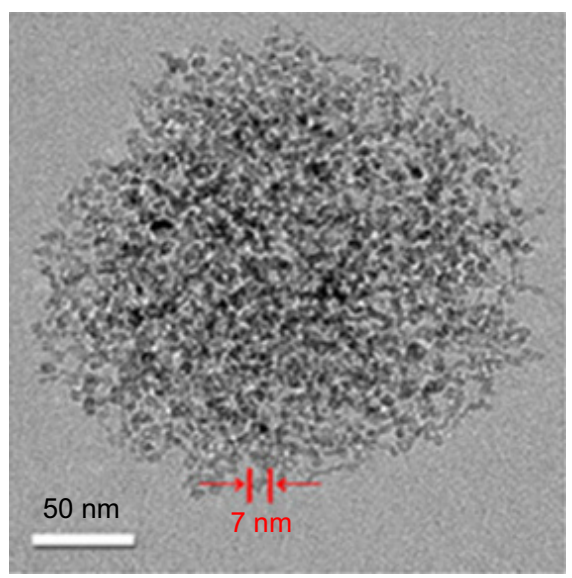

E

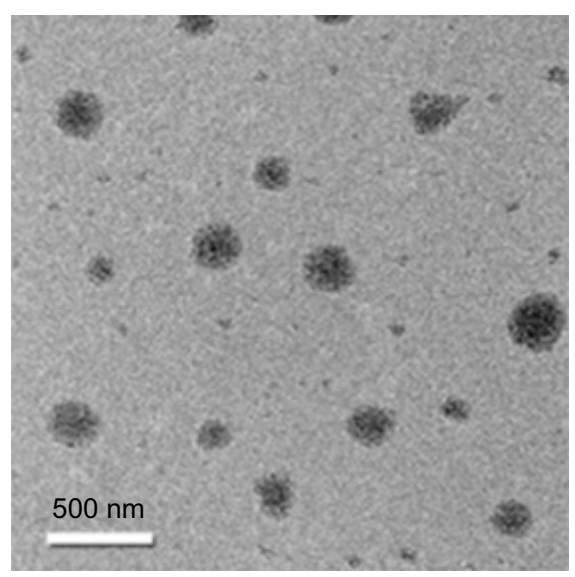

C

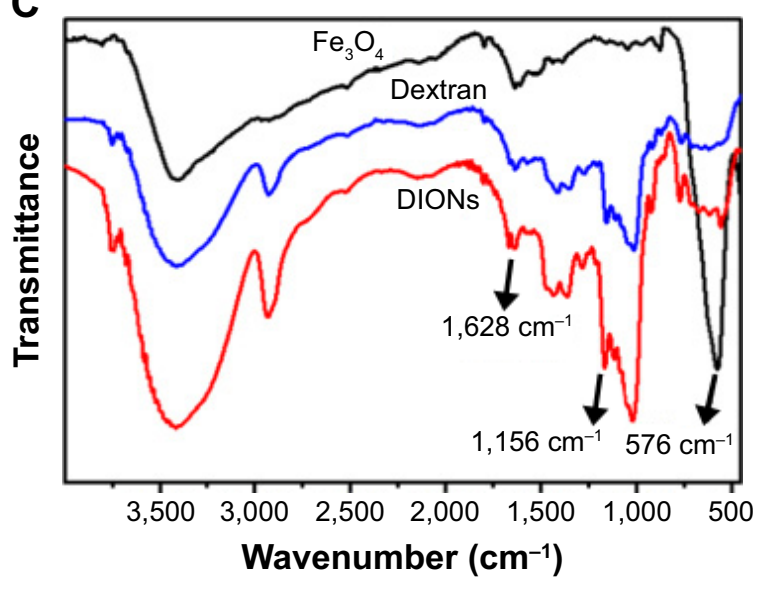

$\mathbf{F}$
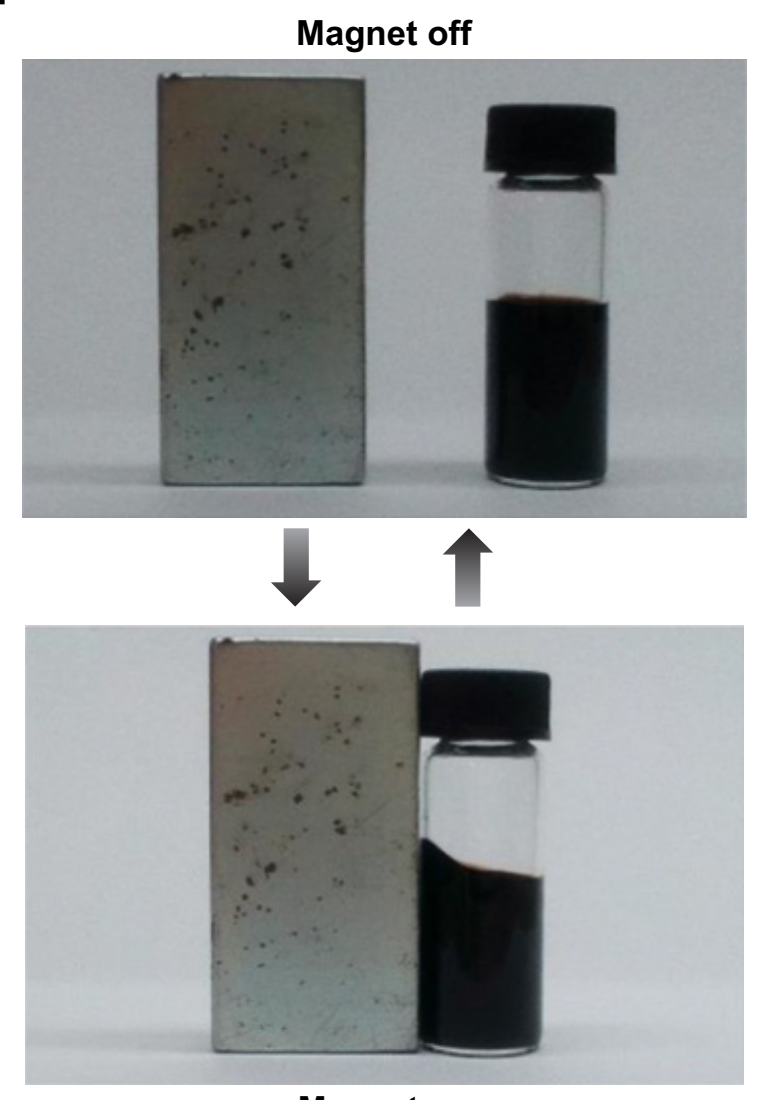

Magnet on

Figure I (A) Facile one-pot fabrication of DIONs. (B) The size and size distribution of DIONs, and (C) FTIR spectra of Fe ${ }_{3} \mathrm{O}_{4}$, dextran and DIONs. (D and E) TEM micrographs. (F) Optical photographs of DIONs solution in the absence and presence of a magnet.

Abbreviations: DIONs, dextran-coated iron oxide nanoclusters; FTIR, Fourier transform infrared; PDI, polydispersity index; TEM, transmission electron microscopy. 
A

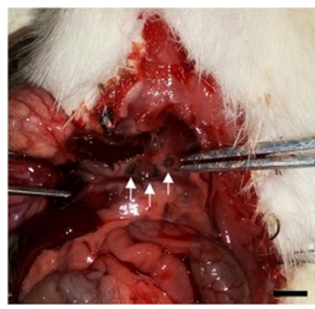

$25 \mathrm{mg} / \mathrm{mL}$

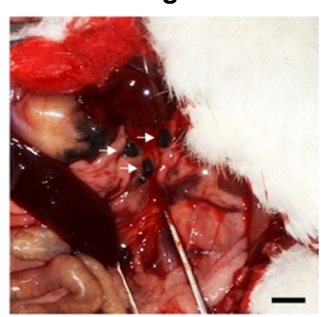

$75 \mathrm{mg} / \mathrm{mL}$

C

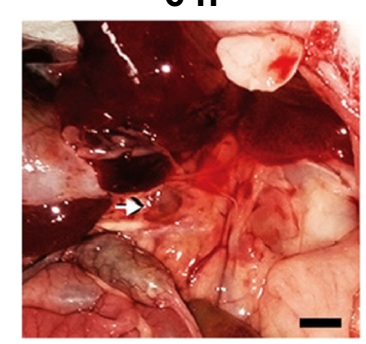

D

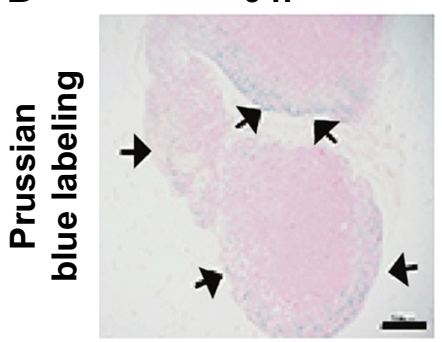

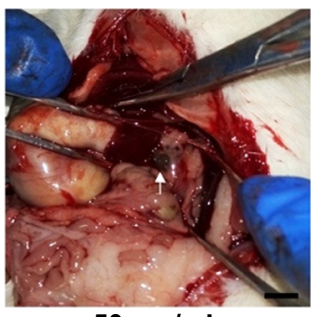

$50 \mathrm{mg} / \mathrm{mL}$

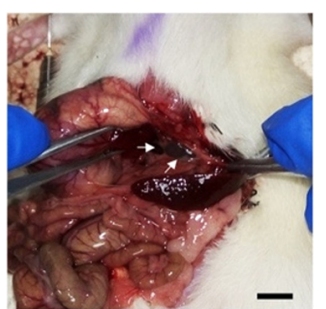

$100 \mathrm{mg} / \mathrm{mL}$

\section{B}
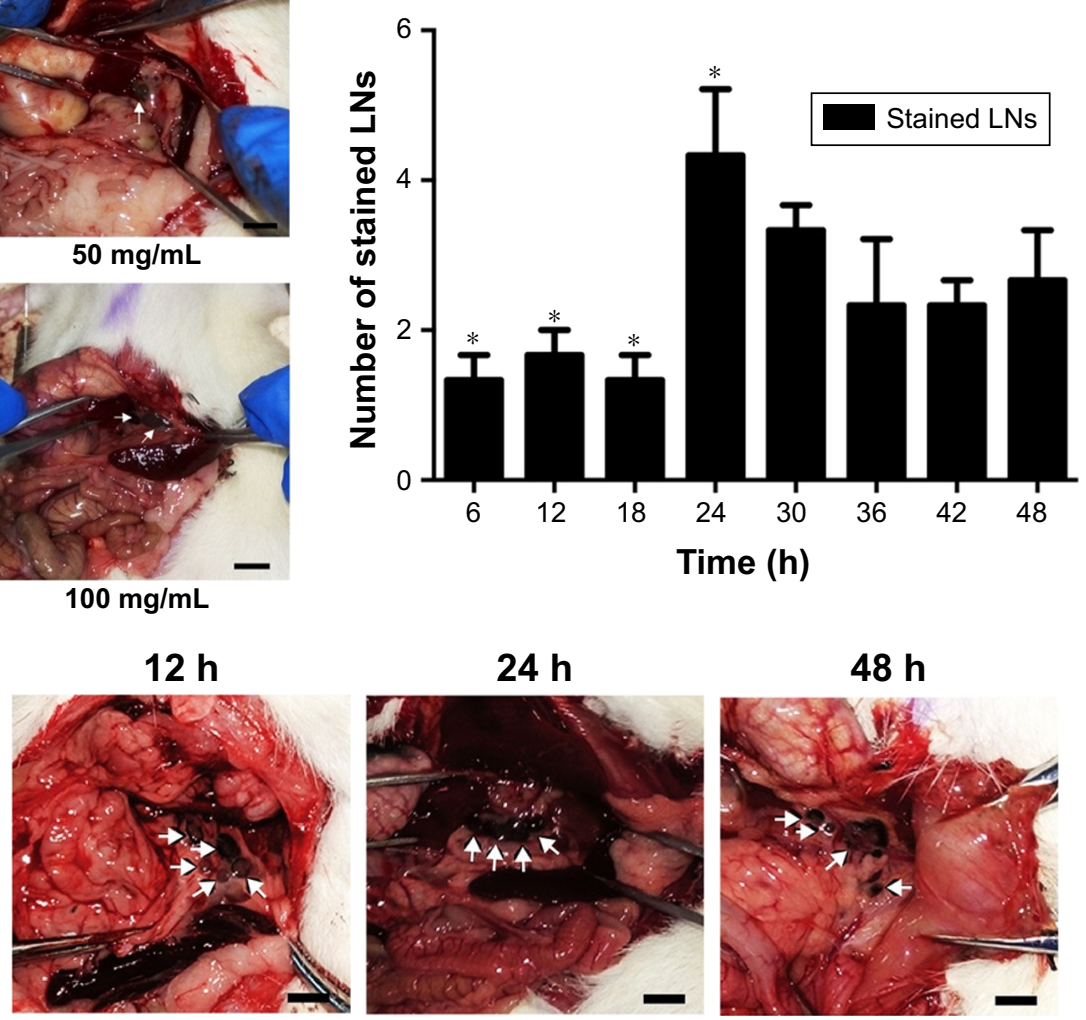

$12 \mathrm{~h}$

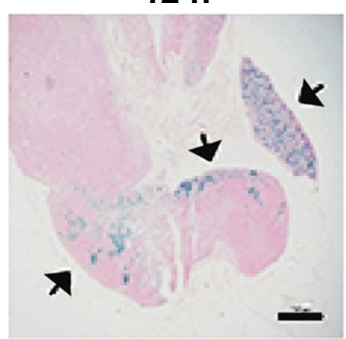

$24 \mathrm{~h}$

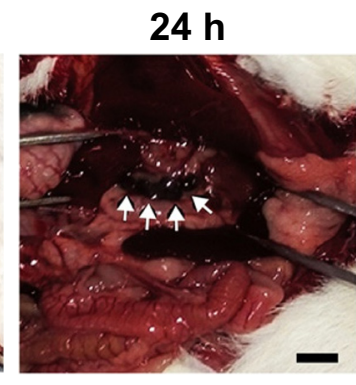

$24 \mathrm{~h}$

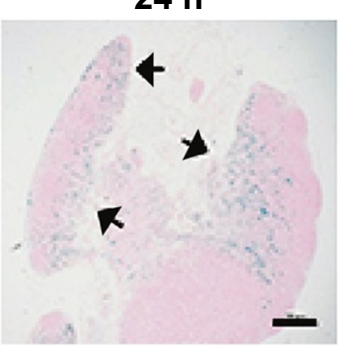

$48 \mathrm{~h}$

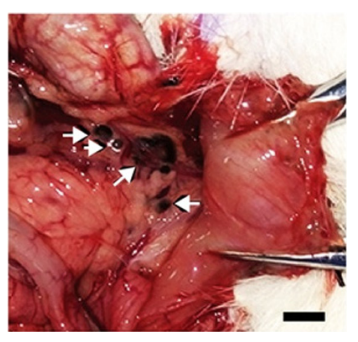

$48 \mathrm{~h}$

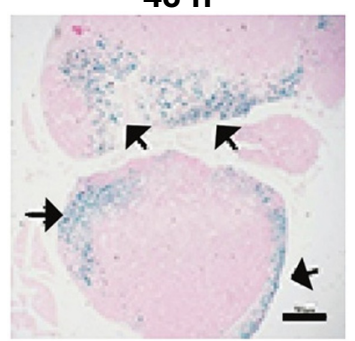

Figure 2 In vivo LNs mapping.

Notes: (A) Different concentrations $(25,50,75$ and $100 \mathrm{mg} / \mathrm{mL})$ of DIONs were injected to explore an appropriate concentration for LN mapping. The scale bars indicate $\mathrm{I} \mathrm{cm}$. (B) The number of stained LNs. The maximum number of stained LNs at $24 \mathrm{~h}(4.33 \pm 1.53, * P<0.05)$. Comparison of numbers at different times indicate statistically significant differences ( $\mathrm{F}=3.354, P<0.05)$. (C) The labeling effect of $75 \mathrm{mg} / \mathrm{mL}$ DIONs from 6 to $48 \mathrm{~h}$. The scale bars indicate I cm. The pictures $(\mathbf{A})$, (B) and (C) were recorded using a Canon digital camera, and white arrows point out the stained LNs. (D) Prussian blue-stained tissue slices of the stained LNs. Black arrows point out the blue stained iron in the LNs. The photos were recorded using a fluorescence microscope (IX-70; Olympus). The scale bars indicate $40 \mu \mathrm{m}$.

Abbreviations: LNs, lymph nodes; DIONs, dextran-coated iron oxide nanoclusters.

layer at 5 points $(0.05 \mathrm{~mL}$ per point $)$ around the primary tumor $24 \mathrm{~h}$ before surgery might have a satisfactory labeling effect and could be used as an efficient molecular imaging probe for accurate, sensitive and specific LN mapping.

\section{In vitro biosafety evaluation}

Despite the abovementioned advantages, biosafety remains a great concern in determining whether DIONs can be used in future clinical studies. ${ }^{22,23}$ To address this issue, in vitro experiments were performed by incubating DIONs with LECs, a dominant cell type that lines the inner surface of the lymphatic vessels in LNs. The isolation, culture and identification of LECs have been described by Dai et al. ${ }^{24}$ The cytotoxicity and proliferation assays were conducted using CCK-8. The results suggested that DIONs had a very low cytotoxicity to cells at different concentrations $(\mathrm{F}=0.684$, $P=0.665$, Figure $3 \mathrm{~A})$. The cell growth curves of normal and DIONs-LECs during a period of $96 \mathrm{~h}$ showed that cell proliferation was obvious, and the comparison of OD values at different times had statistically significant differences $(\mathrm{F}=494.101, * * P<0.001)$ but had no significant difference between the 2 groups ( $\mathrm{F}=0.429, P=0.787$; Figure $3 \mathrm{~B})$. So the 
A

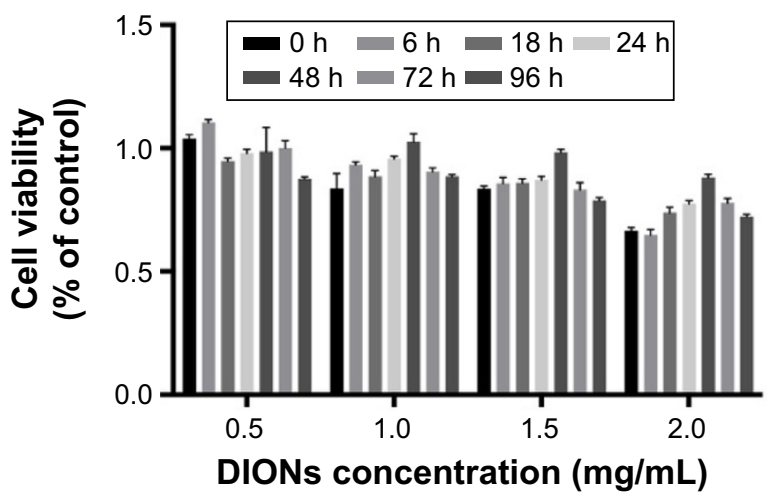

B

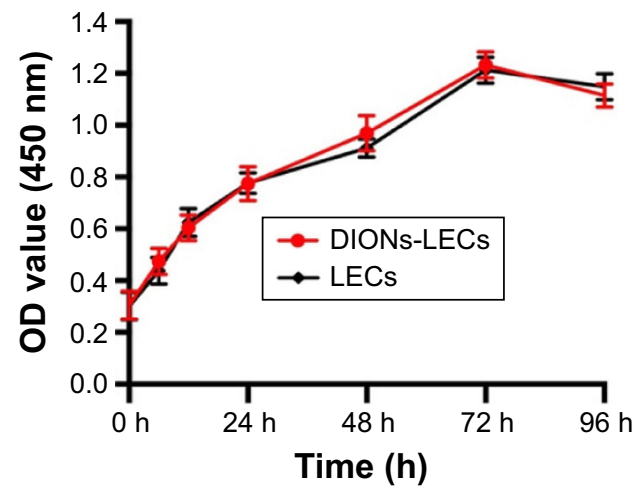

C

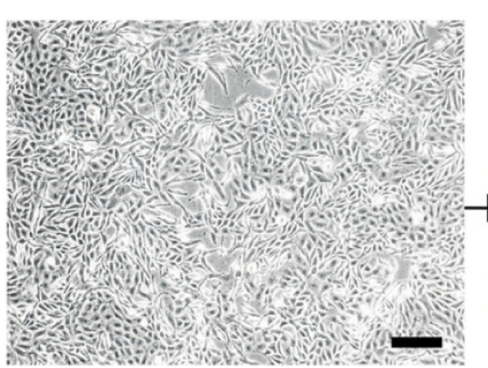

DIONs-LECs

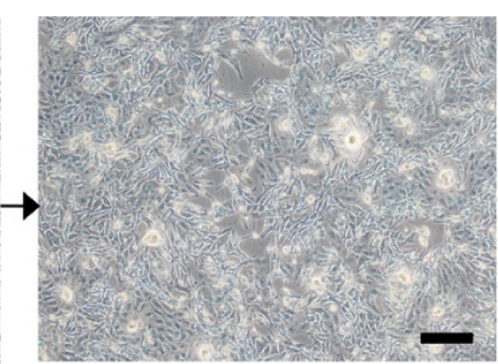

Prussian blue labeling

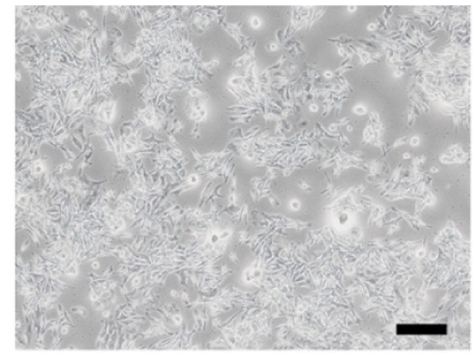

LECs without the DIONs

Figure 3 In vitro biosafety evaluation.

Notes: (A) Cell viability (\% of control) of LECs after coincubation of DIONs with different concentrations $(0.5,1.0,1.5 \mathrm{and} 2.0 \mathrm{mg} / \mathrm{mL})$ for $0 \mathrm{~h}$ to $96 \mathrm{~h}(\mathrm{~F}=0.684, \mathrm{P}=0.665)$; (B) cell growth curves of normal and DIONs-LECs during a period of $96 \mathrm{~h}$ showed that cell proliferation was obvious ( $\mathrm{F}=494$. I0I, $\mathrm{P}<0.00 \mathrm{I})$, but there was no significant difference between the 2 groups $(F=0.429, P=0.787)$; (C) optical images of LECs-DIONs that were stained with Prussian blue to verify the presence of the DIONs. These were observed using a fluorescence microscope (IX-73, Olympus). The scale bars indicate $100 \mu \mathrm{m}$.

Abbreviations: LECs, lymphatic endothelial cells; DIONs, dextran-coated iron oxide nanoclusters; OD, optical density.

CCK-8 assay showed that DIONs had a very low cytotoxicity to cells and no visible effect on proliferation. Next, LECs were coincubated with $0.5 \mathrm{mg} / \mathrm{mL}$ of DIONs for $24 \mathrm{~h}$ to show that DIONs had little influence on the morphology of the LECs (Figure 3C). All these in vitro data demonstrated that DIONs had good biocompatibility and biosafety at the cellular level.

\section{Evaluation of in vivo biodistribution}

In this experiment, Sprague Dawley rats were injected with DIONs-Cy5 to evaluate the biodistribution of these particles during the LN mapping period. The fluorescent images were captured at $6,12,24$ and $48 \mathrm{~h}$ by a Carestream imaging system. After $48 \mathrm{~h}$, the treated rats were sacrificed with a pentobarbital overdose, and their major organs (liver, spleen, kidney, heart, lung and LNs) were excised and imaged with a small animal in vivo fluorescence imaging system. The ex vivo fluorescence images indicate that the DIONs-Cy5 assemble in the LNs, except around the gastric injection points. Furthermore, only a small amount of fluorescence could be found in the heart, lung, spleen and other important metabolic organs, such as the liver and kidney, which further validates that DIONs only access the lymphatic vessels instead of entering the bloodstream after submucosal injection (Figure 4A and B).

\section{Evaluation of in vivo biosafety}

At the third month, a long-term tracing in vivo (4 days, 1 week, 2 weeks, 1 month, 2 months and 3 months) revealed that there was less nanoparticle residue around the injection point in the DIONs group than in the CNPs group (Figure 5A). Furthermore, the DIONs group had no complications, such as acute inflammatory exudation, gastric perforation or adhesive intestinal obstruction, etc., or any abnormal behaviors, compared with the control group (Figure 5B). The histological examination further showed that the major organs (liver, kidney, spleen, heart and brain) of the DIONs group had no appreciable pathological lesion at the 5 time points ( 4 days, 1 week, 2 weeks, 1 month, 2 months and 3 months), which indicated that the DIONs might not be catabolized in these major organs by blood circulation or through the blood-brain barrier (Figure 5C). In addition, to quantitatively evaluate and compare the long-term toxicity of DIONs and CNPs, the blood biochemistry indices, including 
A

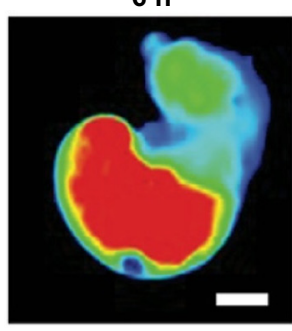

$12 \mathrm{~h}$

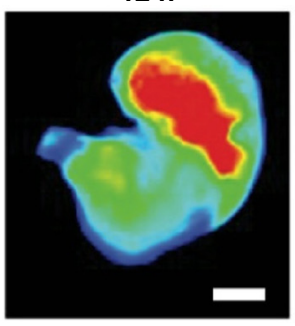

$24 \mathrm{~h}$

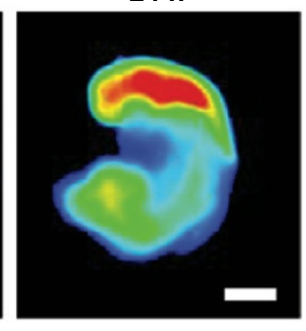

$48 \mathrm{~h}$

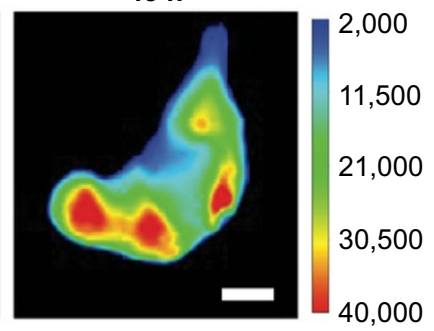

B

B Liver

Heart

\begin{abstract}
Lung
\end{abstract}
Kidney

Spleen

LNs
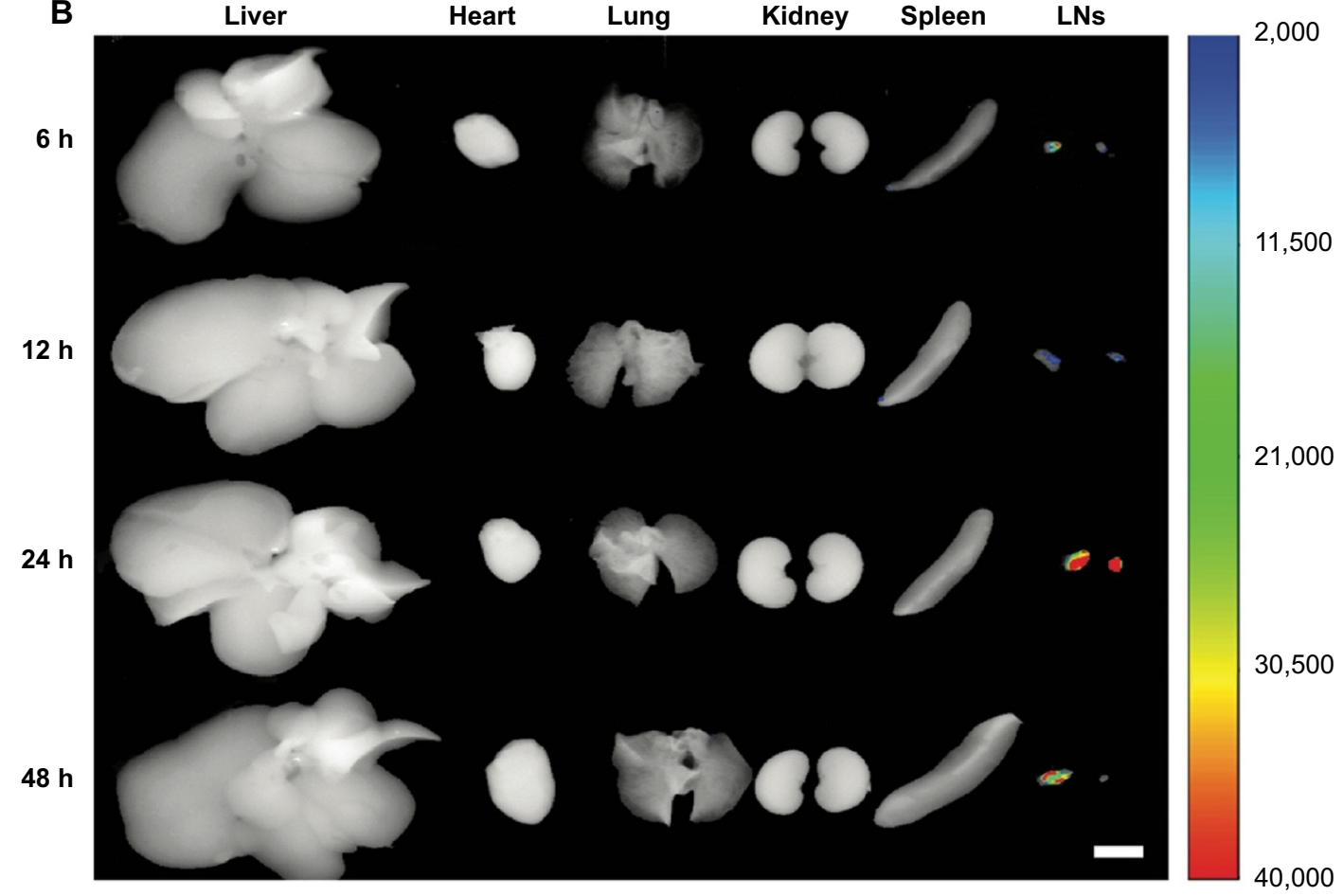

Figure 4 Evaluation of in vivo biodistribution.

Notes: (A) The dynamic distribution of drugs after rats' gastric submucosal layer was injected from $6 \mathrm{~h}$ to $48 \mathrm{~h}$. (B) The rats' perigastric LNs and major organs were excised and imaged. Images produced by in vivo fluorescence imaging system (Carestream In-Vivo Imaging System FXPRO). The scale bars indicate I cm. Abbreviation: LNs, lymph nodes.

albumin (ALB), alanine transaminase (ALT), aspartate transaminase (AST), blood urea nitrogen (BUN) and creatinine (CREA), were measured. Among the abovementioned indices, the ALT and AST indices are closely related to the liver function of animals, while CREA and BUN levels relate to kidney function. ${ }^{18}$ The results revealed that there were no significant differences in any of the indices of the treated rat compared to the untreated rat within an observation period of 3 months, which proved that the DIONs might have no harmful impact on liver and kidney function (Figure 5D). In order to further evaluate the biosafety, the testing of the blood iron content was performed. At 6, 12, 24, 48, 96 and $168 \mathrm{~h}$ after injection, the blood iron content exhibited no significant changes between the DIONs group and the normal group (F=4.005, $P=0.060$; Figure 5E). These results showed that few DIONs spread into the blood circulation after the local injection. All the in vivo results suggested that DIONs have good biosafety for LN mapping.

\section{Conclusion}

The current study testified that DIONs could serve as a simple but efficient system for LN mapping. Furthermore, a longterm dynamic investigation ( 3 months) in vivo revealed the satisfactory biosafety and stability for LN mapping, which could be attributed to proper particle size and the uptake of DIONs by LECs. In addition, both in vitro and in vivo evaluation results indicated that DIONs had acceptable biocompatibility and biosafety, which may facilitate direct assessment of the LNs in gastric cancer staging. In addition, our recent preliminary studies further demonstrated that DIONs could 
A

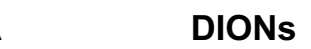

Prussian blue labeling

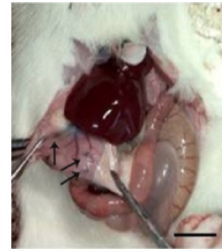

C
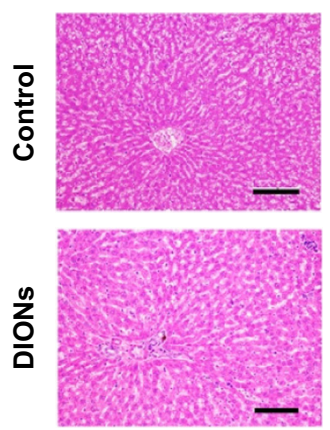

D
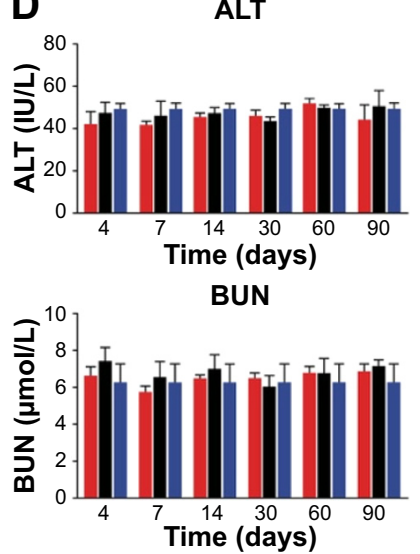

\begin{tabular}{|l|}
\hline DIONs a CNPs $\quad$ Control \\
\hline
\end{tabular}
CNPs

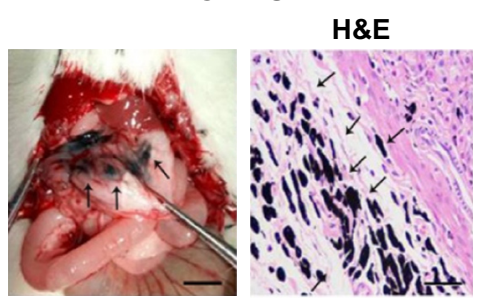

Kidney

Spleen
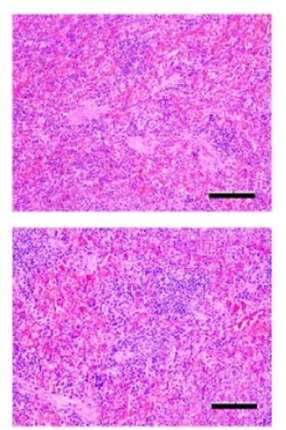

AST

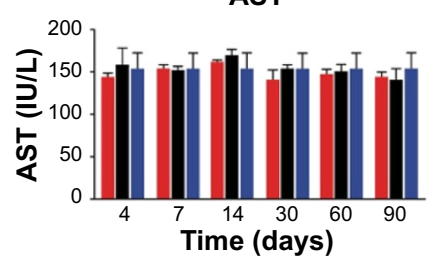

CREA

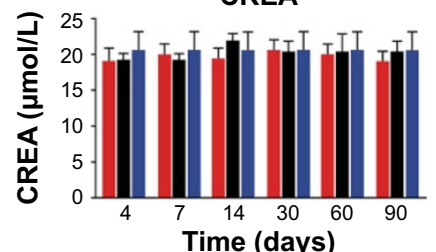

Time (days)
B

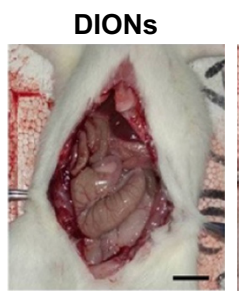

Control

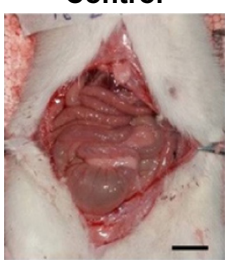

Brain
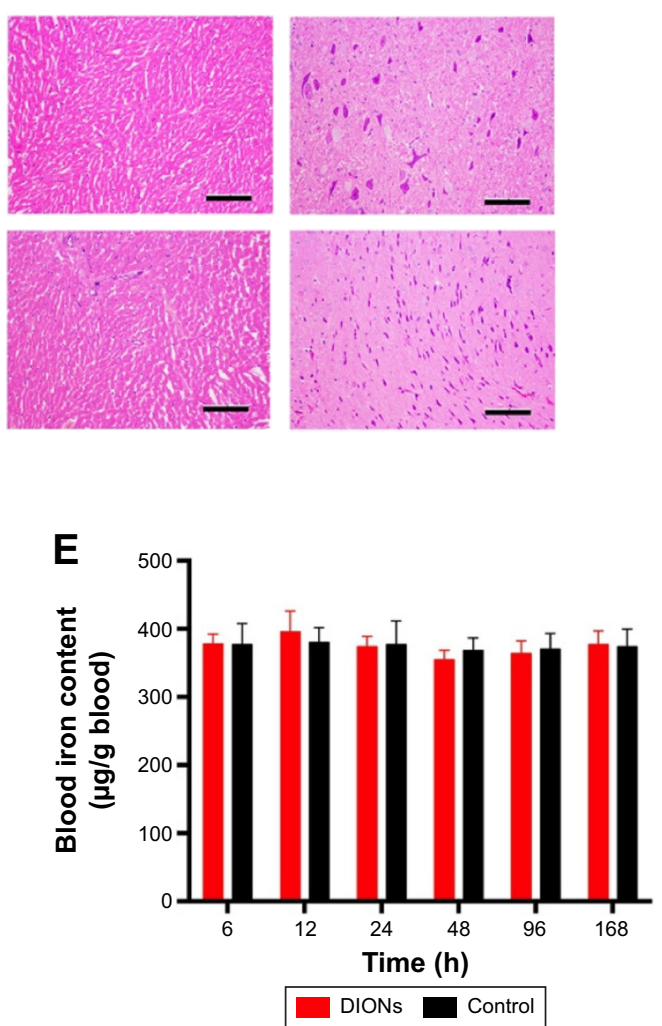

Figure 5 In vivo biosafety evaluation.

Notes: (A) Drug residues around the injection point of DIONs and CNPs at the third month. Black arrows indicate the drug residues around the injection point and the scale bars indicate $\mathrm{I} \mathrm{cm}$. In the same way, black arrows indicate the drug residues in the Prussian blue labeling and $\mathrm{H} \& \mathrm{E}$ picture, and the scale bars indicate $400 \mu \mathrm{m}$. (B) Postoperative complications in the DIONs group compared with the control group. The scale bars indicate I cm. (C) Pathological lesions in the major organs evaluated by H\&E, the scale bars indicate $200 \mu \mathrm{m}$. (D) The blood biochemistry for liver and kidney function. Influence of DIONs on blood biochemistry indices of the treated rats at 4 days, I week, 2 weeks, I month, 2 months and 3 months. ALT ( $F=2.238, P=0.086)$, AST ( $F=0.83, P=0.535), B U N(F=0.4 I 5, P=0.836)$ and $C R E A(F=0.3, P=0.9 I 2)$. ( $E)$. The testing of the blood iron content from $6 \mathrm{~h}$ to $168 \mathrm{~h}(\mathrm{~F}=4.005, P=0.060)$.

Abbreviations: DIONs, dextran-coated iron oxide nanoclusters; CNPs, carbon nanoparticles; H\&E, hematoxylin and eosin; ALT, alanine transaminase; AST, aspartate transaminase; BUN, blood urea nitrogen; CREA, creatinine.

also serve as a safe and effective material carrier for tumorspecific tracers and targeted chemotherapy.

\section{Acknowledgments}

This research was supported by the Major Program of the Science and Technology Program of Guangzhou (no 201508020047), 973 Program of China (no 2015CB755500) and the National Natural Science Foundation of China (nos 51273216, J1103305), President Foundation of Nanfang
Hospital, Southern Medical University (no 2016B021) and Special Funds for Public Welfare Research and Capacity Building of Guangdong Province in China (no 2014A010105025). The authors appreciate the technical support and other help from Mrs Tingting Li, Mr Baoxiong Zhuang, Mr Gengtai Ye and Mr Yuming Jiang.

\section{Disclosure}

The authors report no conflicts of interest in this work. 


\section{References}

1. Niu C, Wang Z, Lu G, et al. Doxorubicin loaded superparamagnetic PLGA-iron oxide multifunctional microbubbles for dual-mode US/MR imaging and therapy of metastasis in lymph nodes. Biomaterials. 2013;34(9):2307-2317.

2. Hu Y, Huang C, Sun Y, et al. Morbidity and mortality of laparoscopic versus Open D2 distal gastrectomy for advanced gastric cancer: a randomized controlled trial. J Clin Oncol. 2016;34(12):1350-1357.

3. Koizumi N, Harada Y, Murayama Y, et al. Detection of metastatic lymph nodes using 5-aminolevulinic acid in patients with gastric cancer. Ann Surg Oncol. 2013;20(11):3541-3548.

4. Wang Y, Deng H, Chen H, et al. Preoperative submucosal injection of carbon nanoparticles improves lymph Node staging accuracy in rectal cancer after neoadjuvant chemoradiotherapy. J Am Coll Surg. 2015; 221(5):923-930.

5. van der Zaag ES, Bouma WH, Tanis PJ, Ubbink DT, Bemelman WA, Buskens CJ. Systematic review of sentinel lymph node mapping procedure in colorectal cancer. Ann Surg Oncol. 2012;19(11):3449-3459.

6. Kwee RM, Kwee TC. Imaging in assessing lymph node status in gastric cancer. Gastric Cancer. 2009;12(1):6-22.

7. Tanaka E, Choi HS, Fujii H, Bawendi MG, Frangioni JV. Imageguided oncologic surgery using invisible light: completed pre-clinical development for sentinel lymph node mapping. Ann Surg Oncol. 2006; 13(12):1671-1681.

8. Yun M, Lim JS, Noh SH, et al. Lymph node staging of gastric cancer using (18)F-FDG PET: a comparison study with CT. J Nucl Med. 2005;46(10):1582-1588.

9. Kim EY, Lee WJ, Choi D, et al. The value of PET/CT for preoperative staging of advanced gastric cancer: comparison with contrast-enhanced CT. Eur J Radiol. 2011;79(2):183-188.

10. Shimada H, Okazumi S, Koyama M, Murakami K. Japanese Gastric Cancer Association Task Force for Research Promotion: clinical utility of ${ }^{18} \mathrm{~F}$-fluoro-2-deoxyglucose positron emission tomography in gastric cancer. A systematic review of the literature. Gastric Cancer. 2011; $14: 13-21$.

11. Kitagawa Y, Fujii H, Kumai K, et al. Recent advances in sentinel node navigation for gastric cancer: a paradigm shift of surgical management. J Surg Oncol. 2005;90(3):147-151. Discussion 151.

12. Chen H, Wang Y, Xue F, et al. [Application of subserosal injection of carbon nanoparticles via infusion needle to label lymph nodes in laparoscopic radical gastrectomy]. Zhonghua Wei Chang Wai Ke Za Zhi. 2014; $17: 457-460$
13. Mehvar R. Dextrans for targeted and sustained delivery of therapeutic and imaging agents. J Control Release. 2000;69(1):1-25.

14. Dang W, Colvin OM, Brem H, Saltzman WM. Covalent coupling of methotrexate to dextran enhances the penetration of cytotoxicity into a tissue-like matrix. Cancer Res. 1994;54(7):1729-1735.

15. Nevozhay D, Budzynska R, Kanska U, et al. Antitumor properties and toxicity of dextran-methotrexate conjugates are dependent on the molecular weight of the carrier. Anticancer Res. 2006;26(2A):1135-1143.

16. Sugahara S, Kajiki M, Kuriyama H, Kobayashi TR. Carrier effects on antitumor activity and neurotoxicity of AZ10992, a paclitaxelcarboxymethyl dextran conjugate, in a rats model. Biol Pharm Bull. 2008;31(2):223-230

17. Kamat M, El-Boubbou K, Zhu DC, et al. Hyaluronic acid immobilized magnetic nanoparticles for active targeting and imaging of macrophages. Bioconjug Chem. 2010;21(11):2128-2135.

18. Jayapaul J, Arns S, Bunker M, et al. In vivo evaluation of riboflavin receptor targeted fluorescent USPIO in mice with prostate cancer xenografts. Nano Res. 2016;9(5):1319-1333.

19. Brabec M, Schober D, Wagner E, et al. Opening of size-selective pores in endosomes during human rhinovirus serotype 2 in vivo uncoating monitored by single-organelle flow analysis. $J$ Virol. 2005;79(2): $1008-1016$.

20. International Organization for Standardization. ISO 8294:1994. Animal and vegetable fats and oils - Determination of copper, iron and nickel contents - Graphite furnace atomic absorption method. Available at: https://www.iso.org/standard/15414.html. Accessed April 13, 2017.

21. Schleich N, Sibret P, Danhier P, et al. Dual anticancer drug/ superparamagnetic iron oxide-loaded PLGA-based nanoparticles for cancer therapy and magnetic resonance imaging. Int J Pharm. 2013; 447(1-2):94-101.

22. Zhang XD, Wu D, Shen X, Liu PX, Fan FY, Fan SJ. In vivo renal clearance, biodistribution, toxicity of gold nanoclusters. Biomaterials. 2012;33(18):4628-4638.

23. Chen Y, Chen H, Shi J. In vivo bio-safety evaluations and diagnostic/ therapeutic applications of chemically designed mesoporous silica nanoparticles. Adv Mater. 2013;25(23):3144-3176.

24. Dai T, Zhou S, Yin C, et al. Dextran-based fluorescent Nanoprobes for sentinel lymph node mapping. Biomaterials. 2014;35(28):8227-8235.
International Journal of Nanomedicine

\section{Publish your work in this journal}

The International Journal of Nanomedicine is an international, peerreviewed journal focusing on the application of nanotechnology in diagnostics, therapeutics, and drug delivery systems throughout the biomedical field. This journal is indexed on PubMed Central, MedLine, CAS, SciSearch ${ }^{\circledR}$, Current Contents ${ }^{\circledR} /$ Clinical Medicine,

\section{Dovepress}

Journal Citation Reports/Science Edition, EMBase, Scopus and the Elsevier Bibliographic databases. The manuscript management system is completely online and includes a very quick and fair peer-review system, which is all easy to use. Visit http://www.dovepress.com/ testimonials.php to read real quotes from published authors. 